\title{
Perfil dos partos cesáreos em um hospital universitário
}

\section{CAESAREAN SECTIONS PROFILE OF A UNIVERSITY HOSPITAL}

\author{
Mariana Costa Hoffmeister ${ }^{1}$, Jessica Oliboni Scapineli ${ }^{1}$, \\ Daniela Akemi Fujita ${ }^{1}$, Débora Elisa Rocha Lunardi ${ }^{1}$, \\ Mariane Boeira Resta1 ${ }^{1}$, Raissa Velasques de Figueiredo', \\ Xana Maito Mendes ${ }^{1}$, Mariza Machado Klück ${ }^{2,3}$
}

\section{RESUMO}

Introdução: A proporção de partos cesarianos no Brasil está muito além da preconizada pela Organização Mundial da Saúde (OMS), o mesmo ocorrendo em um Hospital Universitário de Porto Alegre, sul do Brasil. A taxa de cesáreas é considerada um indicador poderoso na avaliação da qualidade da assistência perinatal. Nosso objetivo foi analisar o padrão dos partos cesarianos e normais em um hospital universitário no período de 2004 a 2012 quanto à média de permanência, faixa etária da parturiente, taxa de infecção relacionada ao parto, tipo de pagador e idade gestacional.

Métodos: Estudo de coorte retrospectivo, observacional, com dados coletados no Sistema de Indicadores de Gestão (IG) de um Hospital Universitário, abrangendo o período de 2004 a 2012.

Resultados: A taxa de cesárea no hospital universitário nos anos analisados foi em média $33,21 \%$. Em relação às cesarianas realizadas nesta instituição durante o período em estudo, observou-se que: há uma maior prevalência de cesáreas em mulheres acima de 40 anos, ocorreu um crescimento de partos cesarianos pré-termo, a média de permanência e taxa de infecção foram superiores em relação às mulheres submetidas ao parto vaginal, e houve predomínio de cesarianas na saúde suplementar quando comparada ao Sistema Único de Saúde.

Conclusão: As elevadas taxas de cesárea no hospital universitário, embora acima do recomendado pela OMS, são justificadas por se tratar de um hospital terciário e estão em conformidade com o padrão observado no país.

Palavras-chave: Gestão de qualidade; gestão em saúde; parto; cesárea

\section{ABSTRACT}

Introduction: Cesarean delivery rates in Brazil are beyond that advised by the World Health Organization (WHO), the same occurring in a university hospital of Porto Alegre, southern Brazil. The rate is considered a powerful indicator in evaluation the perinatal quality of care. Our goal was to analyze the pattern of vaginal birth and cesarean delivery rates at a university hospital in the period of 2004-2012 in terms of the average length of stay, mother age, birth-related infection rate, payer type, and gestational age.

Methods: Observational retrospective cohort study with data collected on the Management Indicators System (GI) of a university hospital of Porto Alegre, from 2004 to 2012.

Results: The caesarean rate in our university hospital in the analyzed years averaged $33.21 \%$. Analysis of cesareans performed in this institution during the study period showed that: there is a higher prevalence of cesarean births in women over 40 years,
Clin Biomed Res. 2015;35(1):35-42

1 Faculdade de Medicina, Universidade Federal do Rio Grande do Sul (UFRGS). Porto Alegre, RS, Brasil.

2 Departamento de Medicina Social, Faculdade de Medicina, Universidade Federal do Rio Grande do Sul (UFRGS). Porto Alegre, RS, Brasil.

3 Comissão de Prontuários, Hospital de Clínicas de Porto Alegre. Porto Alegre, RS, Brasil.

\section{Autor correspondente:}

Mariana Costa Hoffmeister

E-mail: mariana.hoffmeister@gmail.com Universidade Federal do Rio Grande do

Sul, Faculdade de Medicina

Rua Ramiro Barcelos, 2400.

90035-003, Porto Alegre, RS, Brasil. 
there was an increase in the number of preterm cesarean sections, length of stay and infection rates in women undergoing cesarean birth were higher than those of women undergoing vaginal birth, and cesarean deliveries predominated in the private health insurance system compared to the Brazilian Unified Health System.

Conclusions: The high rates of cesarean section in our university hospital, although above those recommended by the WHO, are in accordance with the pattern observed in the country and may be explained by the fact that the institution is a tertiary hospital.

Keywords: Quality management; health management; section; caesarean

Os sistemas de informação em saúde podem monitorar o processo de assistência à saúde e aumentar a qualidade da assistência ao paciente. Como a medicina aumenta a sua complexidade, a informática médica é um agente indispensável para a descentralização e a integração'.

A definição de qualidade em saúde é complexa, e sua avaliação não se baseia exclusivamente em termos técnicos ou na prática específica da assistência, mas sim na conformidade ou adequação a um grupo de expectativas ou padrões e em um conjunto de fatores que envolvem elementos individuais e coletivos no estabelecimento desse juízo de valor. Para fins de avaliação da qualidade assistencial, são utilizados indicadores, ou seja, variáveis que medem quantitativamente as variações no comportamento dos critérios de qualidade estabelecidos e proporcionam as informações necessárias e mensuráveis para descrever tanto a realidade como as modificações devidas à presença do serviço ou assistência².

A taxa de cesárea (Equação 1) é tradicionalmente calculada tanto em nosso hospital universitário, localizado na cidade de Porto Alegre, como em outros hospitais e secretarias de saúde, sendo considerada um indicador poderoso na avaliação da qualidade da assistência perinatal. A adoção desse indicador tem por objetivo avaliar a correção da indicação da cesariana, fato que se relaciona diretamente à morbidade materno-infantil. Como definição, a taxa de cesárea é o percentual mensal de procedimentos cirúrgicos do tipo cesariana em relação ao total de parturientes atendidas.

Taxa de Cesárea $=$

( $\Sigma$ Qt cesáreas/ $\Sigma$ Qt parturientes $) \times 100$

A intensificação das intervenções no parto ocorreu em todo mundo e, ainda no fim da década de 80 , já havia uma preocupação dos pesquisadores quanto ao crescimento da taxa de cesárea ${ }^{3}$. Desde 1985, a Organização Mundial da Saúde (OMS) preconiza taxas de cesárea entre $5 \%$ e $15 \%$, não havendo justificativa para taxas maiores que essa ${ }^{4}$. Há limites percentuais estabelecidos segundo a Portaria Técnica/GM $n^{\circ} 466$, de 14 de maio de
2000 para a realização de cesarianas por estado, assim como valores progressivos, com teto de $25 \%$, para todos os estados do Brasil ${ }^{5}$. A justificativa para percentuais altos pode ser a taxa de partos de alto risco, geralmente concentrados em regiões com hospitais terciários e nas cidades com unidades de referência de assistência à gestante em trabalho de parto.

No modelo de atenção adotado no Brasil, o parto é definido como evento médico ou tecnológico; nesse modelo, a gestante é tratada como paciente, e a maior parte dos nascimentos são hospitalares, sendo o médico o profissional responsável pela sua execução ${ }^{6}$. A taxa de partos cesáreos, que era de $38 \%$ em 2000 , cresceu para $52 \%$ em 2010 . Em $2013,16,45 \%$ das internações hospitalares no Brasil se deveram a partos, sendo $41 \%$ cesáreas $^{7}$. Como explicação para esses altos índices de cesárias, Faúndes e Cecatti ${ }^{8}$ descreveram fatores associados à mulher (medo da dor, busca da integridade vaginal e crenças de que o parto vaginal é mais arriscado para o feto do que uma cesárea), organização da atenção obstétrica (conveniência e segurança do médico), fatores institucionais e legais, como o pagamento mais elevado para a cesárea por parte do Instituto Nacional de Assistência Médica e Previdência Social, que por anos foi responsável pelo pagamento dos partos no Brasil até ser extinguido, e, durante certo período, a esterilização cirúrgica durante o procedimento operatório da cesárea ${ }^{9}$.

Assim como em outros países, no Brasil diversas mulheres saudáveis com muito baixo risco de complicações obstétricas por parto vaginal optam por realizar cesárea eletiva. O objetivo final da atenção pré-natal deveria ser chegar a uma decisão de alta qualidade, realizada em conjunto entre a gestante e o médico assistente, que tem conhecimento das melhores evidências disponíveis, sem deixar de se basear nos valores dos pacientes, além das indicações de cesariana clássicas preconizadas na prática médica ${ }^{10}$. Desproporção cefalopélvica $(32,4 \%)$, cesariana prévia $(13,8 \%)$, condição fetal não tranquilizadora $(13,1 \%)$, má apresentação fetal $(11,7 \%)$, gestação múltipla $(0,9 \%)$ e suspeita de 
macrossomia fetal estão entre as indicações mais comumente adotadas para realização de parto cesáreo atualmente ${ }^{11}$. O diagnóstico de desproporção cefalopélvica é difícil por ser dinâmico, só podendo ser feito de forma confiável durante o trabalho de parto. No caso das mulheres com cesariana prévia, há o medo de que ocorra ruptura uterina por haver mais casos de placenta prévia, a maior chance de repetição da indicação anterior e a motivação da paciente para repetir o procedimento. A cardiotocografia só deve ser realizada em gestações de alto risco, pois em gestações de baixo risco aumenta a quantidade de cesarianas sem melhora dos desfechos perinatais. A versão cefálica externa nos fetos em apresentação pélvica é uma medida custo efetiva quando realizada antes do trabalho de parto e a partir de 36 semanas para reduzir pela metade a taxa de cesariana. Nas gestações múltiplas, dependendo da experiência do obstetra e do consentimento materno, o parto pode ser vaginal se o primeiro feto estiver em posição cefálica, mesmo que o próximo não esteja. A macrossomia fetal aumenta o risco de distócia de ombro, com risco maior nos fetos de gestantes diabéticas, embora em $50 \%$ dos casos não se identifique a causa. A indicação de cesárea é profilática devido aos riscos cumulativos de distócia devido ao diabetes materno e à macrossomia.

Em mulheres que já realizaram parto cesáreo, são candidatas à tentativa de parto vaginal as que não tiverem contraindicações ao trabalho de parto, apenas uma incisão transversal baixa e ausência de outras cicatrizes uterinas ou ruptura prévia, pelve adequada, e hospital estruturado para cesariana de emergência ${ }^{11}$.

Atingir as taxas recomendadas pela OMS torna-se, então, um desafio, pois envolve um equilíbrio entre realizar cesáreas apropriadamente indicadas e, ao mesmo tempo, evitar intervenções desnecessárias que não proporcionam melhores desfechos e que podem causar complicações para a mãe e para a criança².

O objetivo deste trabalho é analisar o padrão dos partos cesarianos e normais em um hospital universitário de Porto Alegre, RS durante o período de 2004 a 2012, avaliando faixa etária da parturiente, idade gestacional, taxa de infecção, média de permanência e tipo de pagador.

\section{MÉTODOS}

Estudo de coorte retrospectivo, de caráter observacional. Os dados reunidos para a análise foram coletados no Sistema de Indicadores de Gestão (IG) do Hospital de Clínicas de Porto Alegre (HCPA) em junho de 2013. Foi realizada também pesquisa bibliográfica com seleção de artigos em base de dados utilizando as palavras-chaves: cesárea, partos Brasil, partos Porto Alegre, média de permanência, taxa de infecção, idade materna, idade gestacional.

\section{RESULTADOS}

\section{Taxa de Cesárea}

A análise da taxa de cesárea em nosso hospital universitário durante o período estudado (figura 1) mostra que, em todos os anos, a taxa se manteve bem acima da preconizada pela OMS, que é de 5 a $15 \%$. No ano de 2007 , observou-se a menor taxa do período $(29,67 \%)$. Nos anos seguintes, houve um crescimento no número de cesarianas e, em 2011 , foi registrada a maior taxa $(37,48 \%)$. Nos anos analisados, a taxa de cesariana média foi de $33,21 \%$.

\section{Faixa Etária}

O grupo de adolescentes grávidas (dos 10 aos 19 anos) teve maior taxa de parto vaginal e menor taxa de cesárea, enquanto no grupo de gestantes de idade mais avançada houve maior taxa de cesárea, conforme vemos ao analisarmos os dados de $2004 \mathrm{e}$ 2012 do HCPA (figura 2). Dos 908 partos realizados em jovens menores de 20 anos em 2004, 22,79\% foram cesarianas, enquanto que, dos 135 partos realizados em mulheres com mais de 40 anos, 51,85\% foram cesarianas. No ano de 2012, a grande diferença encontrada entre os grupos se manteve: $25,91 \%$ dos partos de adolescentes foram realizados por meio de cesariana e $50,53 \%$ dos partos de mulheres com mais de 40 anos.

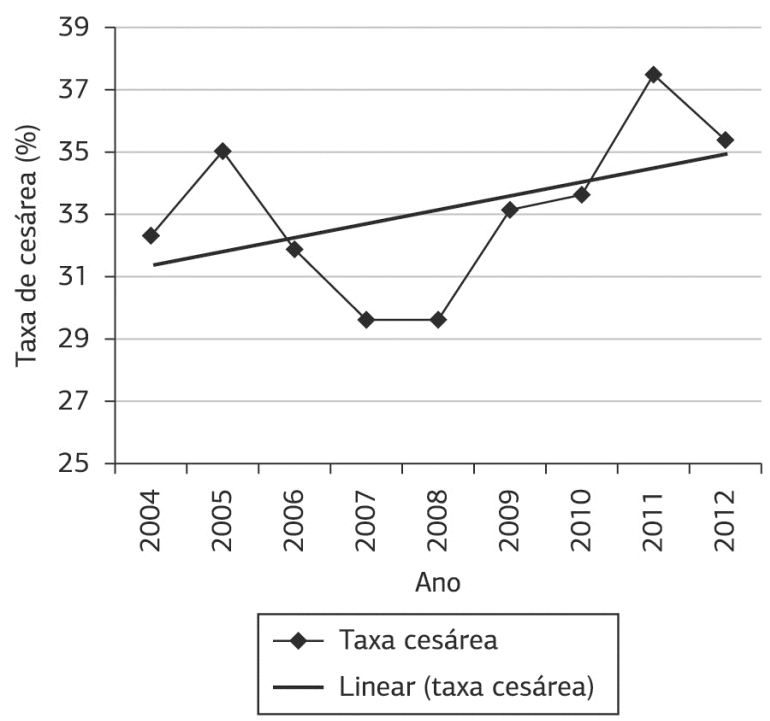

Figura 1: Taxa de cesárea no HCPA de 2004 a 2012. 


\section{Idade Gestacional}

Foram analisadas as taxas de cesariana em nosso hospital universitário nos anos de 2007 e 2012 conforme a idade gestacional da parturiente (figura 3). O início da coleta de dados foi a partir de 2007 por inexistência de registros anteriores no sistema de indicadores. A gravidez a termo, em média, de 40 semanas (normal entre 37 e 41 semanas) teve uma taxa de $40 \%$, semelhante entre os anos analisados. A partir de 2000, há uma tendência ascendente para prematuridade em crianças nascidas por cesárea e uma leve tendência de redução nas crianças nascidas de parto normal ${ }^{8}$.

Quanto à gravidez pré-termo no HCPA, isto é, com menos de 37 semanas, houve um pico da taxa de cesáreas em 2007 entre as semanas 28 e $31(83,72 \%)$ e um pico no ano de 2012 entre as semanas 24 a $27(68,75 \%)$. Em 2012, os partos cesáreos alcançaram a proporção de $33,97 \%$ nos casos de prematuridade extrema (até 31 semanas de gestação), e de $47,08 \%$ nos casos de prematuridade tardia (34 a 36 semanas de gestação), dados semelhantes aos de 2007: 35,46\% e 43,95\%, respectivamente.

\section{Taxa de Infecção}

Foi observado que a taxa de infecção pós-parto em mulheres submetidas a cesariana foi maior do que naquelas que realizaram parto normal em todos os anos analisados. Houve um pico na incidência de infecção relacionada ao parto cesárea no ano de 2007 , chegando a 4,08\%, enquanto a infecção relacionada ao parto normal neste mesmo ano foi de $0,88 \%$. No ano de 2010 , percebeu-se um pico na taxa de infecção relacionada ao parto normal, que chegou a $1,26 \%$, e uma queda importante da taxa relacionadas ao parto cesárea, que foi de 1,99\% (menor taxa desde 2004). Entretanto, essa queda não foi mantida e logo houve um novo aumento, com taxas de 2,5\% em 2011 e 3,13\% em 2012, como mostrado na Figura 4.

\section{Média de Permanência}

Ao analisar dados dos anos de 2007 a 2012 em nosso hospital universitário, constatou-se que há relação direta entre o procedimento cirúrgico e a maior permanência no hospital (figura 5). A média de permanência foi em média de 2,8 dias quando houve parto normal e de 3,95 dias quando relacionada à cesárea. Em todo período observado a média de permanência foi maior entre as puérperas submetidas a cesariana.

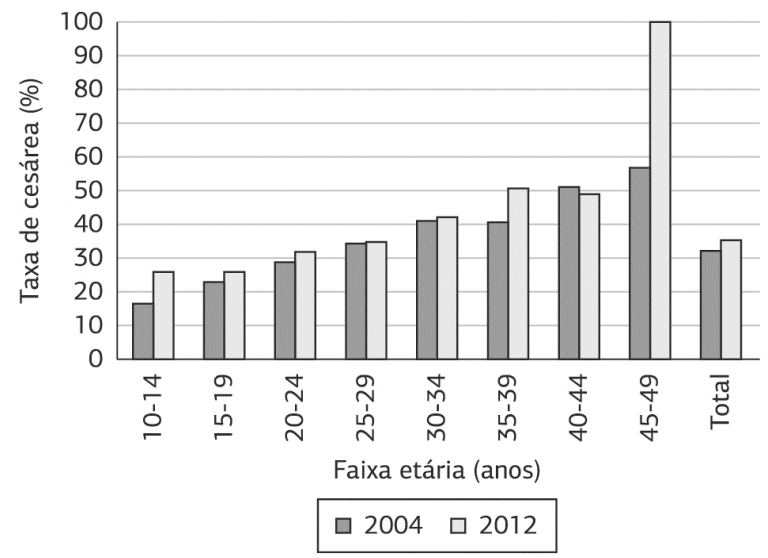

Figura 2: Taxa de cesárea no HCPA em 2004 e 2012 por faixa etária.

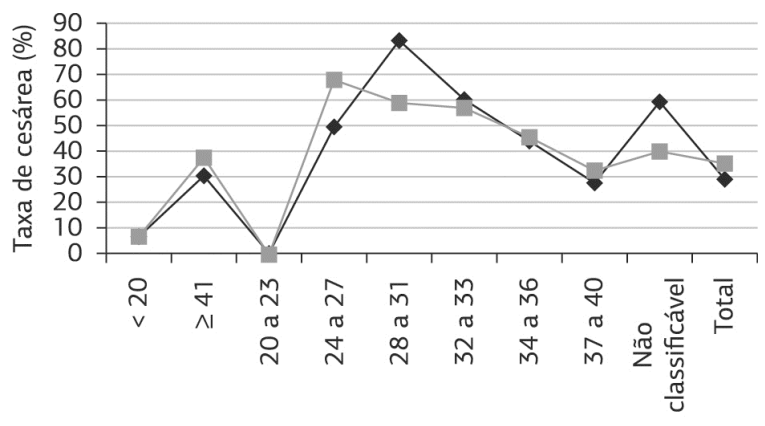

Idade gestacional (semanas)

$$
2007-2012
$$

Figura 3: Taxa de cesárea no HCPA em 2007 e 2012 conforme a idade gestacional.

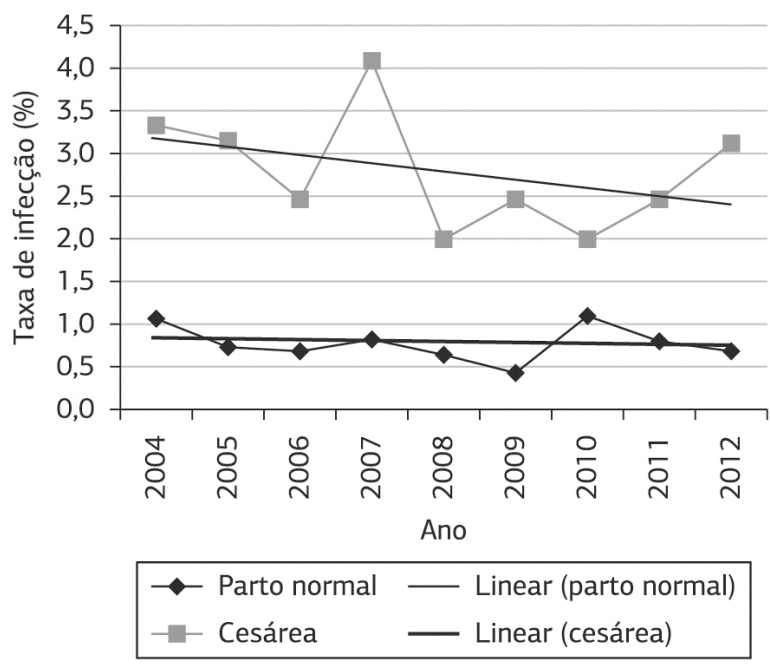

Figura 4: Taxa de infecção relacionada ao tipo de parto no HCPA de 2004 a 2012. 


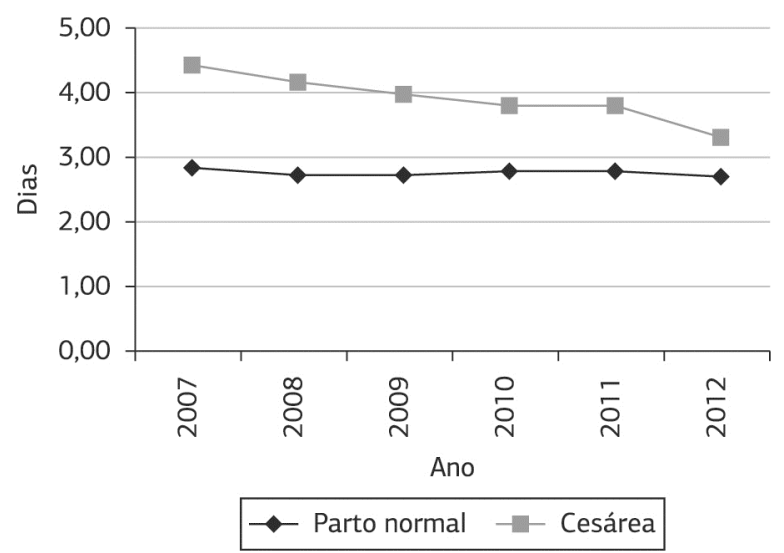

Figura 5: Média de permanência conforme o tipo de parto no HCPA entre 2004 e 2012.

\section{Tipo de Pagador}

No ano de 2004 a taxa de cesáreas pelo Sistema Único de Saúde estava em torno de $30 \%$, já por outros pagadores (particular e convênios) chegava a $60 \%$. Em 2012, a taxa de cesarianas pelo Sistema Único de Saúde, que atende a maior parte da população brasileira ${ }^{12}$, não teve grande aumento em relação a 2004 , mantendo-se abaixo de $40 \%$, mas os outros pagadores apresentaram um salto, chegando próximo a $90 \%$.

\section{DISCUSSÃO}

Vários estudos identificaram inversão do tipo de parto, passando de vaginal para cesáreo, o qual atingiu nos anos 90 a frequência de $53,4 \%$. Mudanças na política de saúde, como o pagamento maior pela cesariana até 1980 implantado pelo Instituto Nacional de Assistência Médica da Previdência Social, influenciaram na escolha do tipo de parto no $\mathrm{Brasil}^{8}$ e podem ter contribuído para o aumento de cesáreas. Mesmo com equivalência do pagamento, a partir da década de 80 , o processo não se reverteu ${ }^{13}$. A laqueadura tubária para esterilização da mulher, realizada durante o parto operatório, tem sido citada como associada ao aumento das cesarianas.

A contribuição da cesariana para melhor assistência médica é indiscutível e constitui opção importante para atender a emergências materno-fetais. No entanto, trata-se de um procedimento não isento de riscos e associado a maior morbimortalidade materno-infantili, 14-16. Um fato que vem sendo observado é que altas taxas de cesárea eletiva (fora da situação de emergência e por outra indicação que não médica) vêm sendo associadas a piores resultados perinatais, e também ao aumento da mortalidade e do risco de infecção, maior uso de antibióticos, mais transfusões, maior tempo de permanência hospitalar após o parto, maior risco de "prematuridade iatrogênica" e maiores custos aos orçamentos da saúde ${ }^{17,18}$. Vários autores questionam os benefícios da cesárea para o feto e sugerem que a diminuição na mortalidade perinatal, identificada desde a década de 70 , foi independente do incremento do parto cesáreo e que esse aumento desenfreado não se justifica ${ }^{15,16}$.

No Brasil, ocorrem cerca de três milhões de nascimentos ao ano, sendo grande parte deles por meio de cesarianas. Mulheres submetidas a cesáreas tiveram cinco vezes mais chance de ter infecção puerperal (entre 2000-2011) ${ }^{9}$. Registrou-se no HCPA, corroborando as taxas nacionais, maior proporção de eventos desfavoráveis quando é realizada a cesárea, com a taxa variando de 1,99 a 4,08 na cesárea e de 0,48 a 1,12 no parto normal. Acredita-se que isso se deva à incisão cirúrgica, ao maior tempo de cirurgia e à maior perda de sangue, que aumentam o risco de infecção. Chama a atenção o fato de que a taxa de partos cesáreos em nosso hospital universitário é muito maior do que a preconizada pela OMS (15\%), atingindo aproximadamente o dobro desse valor. Entretanto, é necessário analisar a população ao se comparar os índices de cesarianas em locais diferentes, pois índices menores são preconizados em serviços de saúde de baixo risco, enquanto índices maiores são aceitos em hospitais como o HCPA, por estarem relacionados ao atendimento de gestações de alto risco ${ }^{11}$. As normas nacionais estabelecem limites percentuais por estado para a realização de partos cesáreos, bem como critérios progressivos para o alcance do valor máximo de $25 \%$ para todos os estados.

Com relação à faixa etária materna, estudos mostram que há um número cada vez maior de adolescentes grávidas e de mulheres que engravidam pela primeira vez depois dos 30 anos. Nos últimos anos, foi observado que no país ocorre um aumento da fecundidade entre as jovens abaixo dos 20 anos. Segundo dados do Ministério da Saúde, 25,7\% dos partos realizados pelo Sistema Único de Saúde em 1999 eram de adolescentes. Enquanto isso, as gestantes com mais de 40 anos representaram em torno de 2 a $3 \%{ }^{19}$. Em 2004, os partos de jovens abaixo dos 20 anos de idade corresponderam a $20,52 \%$ dos partos realizados em nosso hospital universitário, enquanto os partos em mulheres com mais de 40 anos corresponderam a 3,05\%. Em 2012, os partos em adolescentes foram cerca de $18,90 \%$ e de mulheres maiores de 40 anos $2,46 \%$ do total de partos realizados nessa instituição. As parturientes adolescentes são um grupo especial, no qual é observado um gradual crescimento das taxas de cesariana. Esse crescimento é preocupante, já que 
estas meninas, em geral nulíparas, deveriam ser manejadas com especial atenção a fim de evitar cicatrizes cirúrgicas uterinas e assim preservar o futuro obstétrico da paciente. Um estudo realizado pelo Serviço de Ginecologia e Obstetrícia do HCPA em 2013 mostrou resultados indicando que os principais fatores determinantes para cesariana primária são condições potencialmente modificáveis. $\mathrm{O}$ aumento das taxas de cesarianas entre mulheres nulíparas pode ser o maior responsável pelo aumento de $50 \%$ das taxas de cesariana primária. É necessário conhecer os fatores mais associados à indicação de cesariana em nulíparas para promover estratégias de redução dessa intervenção, pois reconhecidamente a via de parto tende a ser repetida na gestação posterior ${ }^{20}$. Em outros estudos brasileiros, foi observado que as taxas de cesárea estão aumentando entre as adolescentes nos últimos anos, o que também é visto ao analisar os dados do presente estudo. Entretanto, ao contrário da tendência brasileira de aumento de cesáreas principalmente entre as adolescentes de 15-19 anos, em nosso hospital universitário observou-se que houve um crescimento maior das cesáreas no grupo entre 10 e 14 anos.

Um estudo realizado em um hospital universitário no Noroeste do Paraná mostrou que, embora as parturientes estivessem na faixa etária adequada para a gestação, o alto percentual de mães adolescentes $(27,1 \%)$ indica a necessidade de maiores estratégias de planejamento familiar para essa faixa etária. O resultado de gestação na adolescência foi próximo ao encontrado num estudo em São Luís, estado do Maranhão (1997-1998) com 29,4\% ${ }^{21}$, porém mais alto que o encontrado para o estado do Paraná, em 2005, que foi de $20 \%{ }^{22}$. Diversos trabalhos mostram, também, que o aumento da idade materna é acompanhado pelo aumento da taxa de cesariana ${ }^{23}$. Comparando os anos de 2004 e 2012, em relação às taxas do HCPA, percebeu-se, dessa forma, que o pico inicialmente se dava entre 45-49 anos, mas depois passou a ocorrer na faixa etária de 15-19 e 35-39 anos em 2012. Apesar dessa diferença, a tendência é de aumento da taxa de cesárea concomitante ao envelhecimento da mulher.

$\mathrm{Na}$ análise da idade gestacional, uma maior prevalência do procedimento entre mães de recém-nascidos prematuros aponta para a ocorrência de "casualidade reversa", onde a utilização excessiva da cesariana como cirurgia eletiva estaria levando a maiores índices de prematuridade, e não vice-versa, fato também relatado em estudo semelhante conduzido no Rio Grande do Sul24.

Segundo Faúndes e Cecatti ${ }^{8}$, a organização atual da atenção obstétrica tem importante papel nas elevadas taxas de cesariana. Os autores destacam o papel da conveniência de uma intervenção programada para o obstetra, a incerteza quanto à possibilidade de hipóxia ou trauma fetal e a falta de preparo da mulher para o parto como os principais fatores ligados à preferência médica na ocorrência de cesarianas ${ }^{10}$. Isto reforça a ideia de que os fatores relacionados à prática médica têm grande influência na decisão pela cesariana. O medo do nascimento do filho e uma experiência obstétrica traumática prévia são as razões mais prevalentes expressadas pelas gestantes que optam por realizar cesárea eletiva sem indicação médica clássica para o procedimento cirúrgico ${ }^{25}$. $\mathrm{O}$ Congresso Americano de Obstetras e Ginecologistas (ACOG), assim como muitos especialistas em ética, diz que os obstetras podem ceder ao pedido de uma paciente para cesárea por decisão materna, embora não haja obrigação legal ou ética para isso. Caso a paciente e o obstetra discordem sobre a via de parto, o ACOG recomenda referenciamento para outro profissional ${ }^{26}$. Para alguns médicos, as cesarianas são uma forma de medicina defensiva, realizadas a fim de evitar processos criminais ${ }^{27}$. Como já sugerido por outros autores, parece haver uma adequação dos horários do obstetra em sua agenda privada ${ }^{8}$, pois a sobrecarga na jornada de trabalho do obstetra faz com que o profissional não disponibilize seu tempo para aguardar o trabalho de parto, favorecendo a decisão pela cesariana, apesar dos apelos das instituições de saúde.

Por outro lado, a parcela atendida pelo Sistema Único de Saúde muitas vezes não tem a chance de escolher o médico que irá atendê-la e, assim, não tem tanto poder de negociação sobre o tipo de parto que prefere e a forma de atendimento que deseja, visto que o parto é atendido por um profissional médico diferente daquele que fez o pré-natal. Essa desvinculação que ocorre na atenção à gestante no Sistema Único de Saúde pode ser considerada um fator contribuinte para a realização de cesáreas, em função da ausência de informações, no momento do parto, sobre a gestação atual e as anteriores ${ }^{28}$. A pequena parte da população que possui plano ou seguro-saúde pode escolher os profissionais médicos que deseja e negociar com eles o tipo de assistência médica que prefere ${ }^{12}$.

Atualmente, quase um quarto dos nascimentos do Brasil ocorre nos serviços hospitalares privados, onde as taxas de cesárea atingem proporções ao redor de $80 \%$. Já no sistema público, que oferece assistência à maioria da população, as taxas de cesárea chegam, em média, a 35\%. Assim, a compreensão da contribuição de fatores sócio-culturais no aumento das taxas de cesariana é crescente, mas ainda não há consenso sobre as melhores soluções para o problema. Como tentativa de solução, países como 
os EUA e o Canadá implantaram medidas como a reestruturação do ensino médico e mudanças na forma de pagamento médico e hospitalar ${ }^{29,30}$.

No Brasil, o Programa de Humanização do Pré-Natal e Nascimento do Ministério da Saúde, lançado em junho de 2000, fundamenta-se no direito à humanização da assistência obstétrica e neonatal como condição primeira para adequado acompanhamento, além de estabelecer critérios para qualificar a assistência e promover o vínculo entre a assistência ambulatorial e o momento do parto cuja efetividade tenha sido comprovada ${ }^{31}$. Entretanto, os resultados dessas medidas ainda não foram amplamente divulgados. Intervenções eficazes, portanto, devem enfocar meios multidisciplinares, como a equipe médica, a organização dos serviços nas maternidades, além de se basear em conhecimento das particularidades sócio-culturais locais. Desta forma, a informação deve chegar também até as mulheres, combatendo, assim, o estigma de entender erroneamente a cesariana como um "bem de consumo", ou utilizada no sentido de favorecer preferencialmente aquelas mulheres com maior acesso socioeconômico ${ }^{32}$.
Além disso, diferentes estudos vêm sugerindo que fatores pessoais, da mulher e de seu ambiente social ${ }^{33,34}$ bem como regionais e institucionais relacionados à organização dos serviços ${ }^{35}$ constituem aspectos não médicos relacionados ao parto no Brasil, influenciando a cultura da prática obstétrica vigente. Assim sendo, nota-se a importância de observar as taxas de cesárea em um hospital, a fim de reduzir os riscos e a morbidade decorrentes desse procedimento.

O hospital universitário cujos dados utilizamos é um hospital referência no atendimento de gestantes e responde por cerca de $30 \%$ de todas as internações obstétricas de Porto Alegre e 39,5\% das internações obstétricas em hospitais públicos da região, atendendo na maior parte, cerca de $90 \%$, pacientes do Sistema Único de Saúde. As elevadas taxas de cesárea desse hospital universitário, embora acima do recomendado pela OMS, são justificadas por se tratar de um hospital terciário. Ademais, essas taxas estão em conformidade com o padrão observado no país.

\section{REFERÊNCIAS}

1. Sigulem D. Um novo paradigma de aprendizado na prática médica da UNIFESP / EPM [tese]. São Paulo: Universidade Federal de São Paulo; 1997.

2. Kluck M. A gestão da qualidade assistencial do Hospital de Clínicas de Porto Alegre: implementação e validação de indicadores. Rev Adm Saúde. 2008;10:97-102.

3. Notzon FC, Placek PJ, Taffel SM. Comparisons of national cesareansection rates. $N$ Engl $J$ Med. 1987;316(7):386-9. http://dx.doi. org/10.1056/NEJM198702123160706. PMid:3807975

4. Appropriate technology for birth. Lancet. 1985;2(8452):436-7. PMid:2863457.

5. Brasil. Ministério da Saúde. Portaria $n^{\circ} 466$, de 10 de maio de 2000. Institui limites percentuais máximos de cesarianas, em relação ao número total de partos realizados por hospital. Diário Oficial da República Federativa do Brasil. 2000 Maio 10.

6. Domingues RM, Santos EM, Leal MC. Aspectos da satisfação das mulheres com a assistência ao parto: contribuição para o debate. Cad Saude Publica. 2004;20(Suppl 1):S52-
62. http://dx.doi.org/10.1590/S0102311X2004000700006. PMid:16636735

7. Brasil. Ministério da Saúde. DATASUS: informações de saúde. Brasília: Ministério da Saúde; 2014. [citado 2014 jul 07]. Disponível em: http://www2.datasus.gov.br/DATASUS/ index.php?area $=0202$.

8. Faúndes A, Cecatti JG. A operação cesárea no Brasil: incidência, tendências, causas, conseqüências e propostas de ação. Cad Saude Publica. 1991;7(2):150-73. http:// dx.doi.org/10.1590/S0102311X1991000200003. PMid:15830039

9. Brasil. Ministério da Saúde. Secretaria de Vigilância em Saúde. Departamento de Análise de Situação de Saúde. As cesarianas no Brasil: situação no ano de 2010, tendências e perspectivas. In Saúde Brasil 2011: Uma análise da situação de saúde e a vigilância da saúde da mulher. Brasília: Editora do Ministério da Saúde, 2012. p. 373-394.

10. Orfali K. Beyond numbers: the multiple cultural meanings of rising cesarean rates worldwide. Am J Bioeth. 2012;12(7):54-6. http://dx.doi. org/10.1080/15265161.2012.680544. PMid:22694038
11. Freitas F, Martins-Costa SH, Ramos JG, Magalhães JA. Rotinas em obstetrícia. 6. ed. Porto Alegre: Artes Médicas; 2011.

12. Patah LE, Malik AM. Models of childbirth care and cesarean rates in different countries. Rev Saude Publica. 2011;45(1):185-94 http://dx.doi.org/10.1590/S003489102011000100021. PMid:21181056

13. Barros FC, Vaughan JP, Victora CG. Why so many caesarean sections? The need for a further policy change in Brazil. Health Policy Plan. 1986;1(1):19-29. http:// dx.doi.org/10.1093/heapol/1.1.19. PMid:10283013

14. Rattner D. Sobre a hipótese de estabilização das taxas de cesárea do Estado de São Paulo, Brasil. Rev Saude Publica. 1996;30(1):19-33. http://dx.doi.org/10.1590/S003489101996000100004. PMid:9008919

15. O'Driscoll K, Foley M. Correlation of decrease in perinatal mortality and increase in cesarean section rates. Obstet Gynecol. 1983;61(1):1-5. PMid:6823339.

16. Minkoff HL, Schwarz RH. The rising cesarean section rate: can it safely be reversed? Obstet Gynecol. 
1980;56(2):135-43. PMid:7393501.

17. Villar J, Valladares E, Wojdyla D, Zavaleta N, Carroli G, Velazco A, et al. Caesarean delivery rates and pregnancy outcomes: the 2005 WHO global survey on maternal and perinatal health in Latin America. Lancet. 2006;367(9525):1819-29. http://dx.doi.org/10.1016/S01406736(06)68704-7. PMid:16753484

18. Belizán JM, Althabe F, Barros FC, Alexander S. Rates and implications of caesarean sections in Latin America: ecological study. BMJ. 1999;319(7222):1397400. http://dx.doi.org/10.1136/ bmj.319.7222.1397. PMid:10574855

19. Ximenes FMA, Oliveira MCR. A influência da idade materna sobre as condições perinatais. Bras Promoção Saúde. 2004;17:56-60.

20. Accetta SG, Salazar CC, Vettorazzi J, Capp E, Oppermann ML, Passos EP. Cesariana primária em nulíparas - fatores de risco em hospital público universitário. Rev HCPA. 2013;33:198204.

21. Simões VM, Silva AA, Bettiol $H$. Lamy-Filho F, Tonial SR, Mochel EG. Characteristics of adolescent pregnancy in São Luís, Maranhão, Brazil. Rev Saude Publica. 2003;37:559-65. PMid:14569330.

22. Brasil. Ministério da Saúde. DATASUS: informações de saúde. Brasília: Ministério da Saúde; 2002. [citado 2007 jul 15]. Disponível em: http://www2.datasus.gov.br/DATASUS/ index.php?area $=0202$.
23. Paim AD, Apolinário EC, Zampieri JF, Kluck MM. Taxa de cesárea primária no Hospital de Clínicas de Porto Alegre. Rev HCPA. 2008;28:136-41.

24. Freitas PF, Drachler ML, Leite JCC, Grassi PR. Desigualdade social nas taxas de cesariana em primíparas no Rio Grande do Sul. Rev Saude Publica. 2005;39(5):7617. http://dx.doi.org/10.1590/S003489102005000500010 . PMid:16254652

25. Kottmel A, Hoesli I, Traub R, Urech C, Huang $D$, Leeners $B$, et al. Maternal request: a reason for rising rates of cesarean section? Arch Gynecol Obstet. 2012;286(1):93-8. http://dx.doi. org/10.1007/s00404-012-2273-y. PMid:22407124

26. Ecker J. Elective cesarean delivery on maternal request. JAMA. 2013;309(18):1930-6. http://dx.doi. org/10.1001/jama.2013.3982. PMid:23652524

27. Minkoff $H$. Fear of litigation and cesarean section rates. Semin Perinatol. 2012;36(5):3904. http://dx.doi.org/10.1053/j. semperi.2012.04.025. PMid:23009974

28. Hotimsky SN, Rattner D, Venancio SI, Bogus CM, Miranda MM. Childbirth as I see it ... or the way I wish it was? Expectations of pregnant women towards childbirth and obstetric care in the public health care system. Cad Saude Publica. 2002;18(5):1303-11. http://dx.doi.org/10.1590/S0102311X2002000500023. PMid:12244363

29. Macfarlane A, Chamberlain G. What is happening to caesarean section rates? Lancet. 1993;342(8878):1005-
6. http://dx.doi.org/10.1016/01406736(93)92874-S. PMid:8105260

30. Millar WJ, Nair C, Wadhera S. Declining cesarean section rates: a continuing trend? Health Rep. 1996;8(1):17-24. PMid:8844177.

31. Tornquist CS. The paradoxes of humanized childbirth care in a public maternity ward in Brazil. Cad Saude Publica. 2003;19(Suppl 2):S419-27. http://dx.doi.org/10.1590/S0102311X2003000800023. PMid:15029361

32. Béhague DP, Victora CG, Barros FC. Consumer demand for caesarean sections in Brazil: informed decision making, patient choice, or social inequality? A population based birth cohort study linking ethnographic and epidemiological methods. BMJ. 2002;324(7343):942-5. http://dx.doi. org/10.1136/bmj.324.7343.942. PMid:11964338

33. Faûndes A, Cecatti JG. Which policy for caesarian section in Brazil? Analysis of trends and consequences. Health Policy Plan. 1993;8(1):3342. http://dx.doi.org/10.1093/ heapol/8.1.33.

34. Barros FC, Vaughan JP, Victora CG, Huttly SR. Epidemic of caesarean sections in Brazil. Lancet. 1991;338(8760):167-9. http://dx.doi. org/10.1016/0140-6736(91)90149-J. PMid:1677075

35. Bailit JL, Love TE, Mercer B. Rising cesarean rates: are patients sicker? Am J Obstet Gynecol. 2004;191(3):800-3. http://dx.doi. org/10.1016/j.ajog.2004.01.051. PMid:15467544 